\title{
Effect of Cationic UV Absorber on Light Fastness Property of Reactive Dye
}

\author{
Zahid LATIF ${ }^{1, a}$, Fan LIU ${ }^{1, b}$, Shu WEN ${ }^{1, c}$, Shao LONG ${ }^{2, d}$, Xin-Yue XIAO ${ }^{3, e}$,
} Li-Na LIN ${ }^{1, f^{*},}$, Ying-Jie CAI ${ }^{1, g}$

${ }^{1}$ Engineering Research Center for Clean Production of Textile Dyeing and Printing, Ministry of Education, Wuhan Textile University, Wuhan 430073, China

${ }^{2}$ Zhejiang Transfer Co., Ltd, Hangzhou 311215, China

${ }^{3}$ Huzhou Angye Digital Industry Co., Ltd, Huzhou 313008, China

azahid.latif786@gmail.com, b837097057@qq.com, '869882975@qq.com, ddawuhan@163.com, ebettyxiao@angyetexdp.com, flinalin@wtu.edu.cn, ${ }^{9}$ yingjiecai@wtu.edu.cn

${ }^{*}$ Corresponding author

Keywords: Dyeing, Cotton, Reactive Dye, Light Fastness, Cationic UV Absorber.

\begin{abstract}
This article focuses on the improvement in light fastness of reactive dyed fabric. Cotton fabric was dyed with Liyuan Yellow F S reactive dye and cationic UV absorber CANFIX SUN was subsequently applied by exhaust method in order to improve light fastness property. The light fastness property of the dyed samples before and after the treatment using UV absorber was tested as per international standards. The results show that for all light, medium and dark shades the dye exhaustion and fixation percentages were very good. In light fastness property, the $\mathrm{dE}$ value (change in colour) of the dyed sample after treatment is smaller in contrasting to that one before treatment which the tone changed to blue and bright direction. It states that this UV absorber improved the light fastness of dyed fabrics.
\end{abstract}

\section{Introduction}

The light fastness of colour is how permanent it is, or how unaffected by light it is. The aversion to, of a material to a change in its colour characteristics as a result of exposure of the material to sunlight or an artificial light source is called light fastness[1]. The colour of a costume or fabric either appeals to us or displeases us more than any other element that enters into it. So, if colour of fabric fades it will lose its attraction during its product life.

The phenomenon by which dyes undergo fading is because of photo degradation is a complex process[2]. Research has shown that $40 \%$ of fading is caused by UV rays. Another $25 \%$ of fading is due to heat, with $25 \%$ being caused by normal visible light. The remaining $10 \%$ cause of fading is from indoor artificial lighting, humidity, and poor dye anchorage. Visible light and UV radiation cause fading. UV light induced uni-molecular decomposition[3] and visible light induced photo oxidation[4]. In all these fading reactions of dyes light in the form of energy and oxygen in the singlet form is required to carry this reaction.

The basic dyes which are famous for their brilliant colors and having poor light fastness are highly light-sensitive when applied on natural fibers. Acid and reactive dyes light fastness is good as compared to basic dyes. All direct dyes do not have good light fastness property but there are some direct dyes having good light fastness property. Vat dyes, such as indigo, are the most lightfast than other types of dyes.

Generally there are two methods to improve light fastness of the colors on a fabric, to choose the dyes having better light fastness property, and/or to apply a UV absorber.[5] The qualities of additive to prove an effective UV protection finish includes excellent absorption of UV radiations at 300-320 nm wavelength and convert this energy into vibrational energy in absorbing dye 
molecule and then leave into environment as thermal energy to avoid photo degradation. Other important requirements are easy application condition and it should not cause any change in color after UV finish application[6, 7].

Thiagarajan and Killi used anti-Oxidants (Gallic acid, Vitamin C and Cafeic acid) and UV absorbers to improve light fastness property of reactive dyes results shows improvement in light fastness in few commercial reactive dyes.[8]

In this research flourene base reactive dye Liyuan Yellow was applied on cotton fabric by exhaust method, which is different in structure than other commercially available reactive dyes. The cotton fabric was dyed with different shade depths to study effect of shade depth on light fastness. This paper explores some possibilities of applying cationic UV absorber to improve light fastness property.

\section{Experimental Procedure}

\section{Materials}

Scoured and bleached plain weaved $100 \%$ cotton fabric was used. Liyuan Yellow F S Reactive dye (commercial grade) was supported by Color Root Hubei Technology Songzi, China, was applied without purification. Commercially available UV absorber CANFIX SUN was sourced from Shanghai Zhanhe Industrial Co. Ltd was used. Sodium chloride and soda ash of laboratory grade were used.

\section{Dyeing}

Scoured and bleached $100 \%$ cotton fabric was dyed by exhaust method at a liquor ratio of 25:1 for different shade depths $0.5,1,3,5$ and $7 \%$ o.w.f according to dyeing profile shown in Fig.1. Different amount of salt and soda ash were used according to dye \% o.w.f (Tab.1).

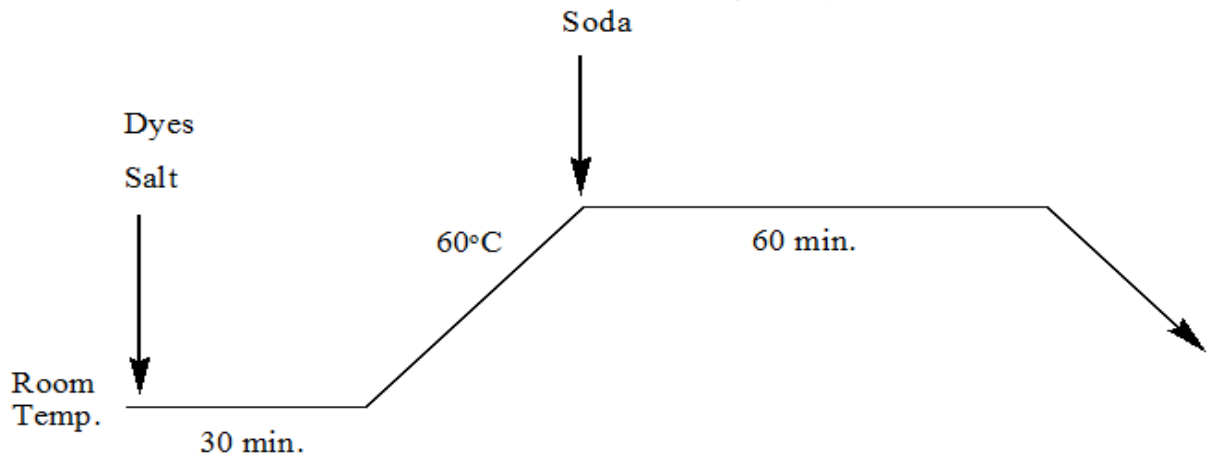

Fig.1 Dyeing profile

Tab.1 Salt and soda ash amount according to dye \% o.w.f

\begin{tabular}{llllll}
\hline Dye o.w.f $(\%)$ & 0.5 & 1 & 3 & 5 & 7 \\
\hline Salt $(\mathrm{g} / \mathrm{L})$ & 40 & 50 & 80 & 100 & 100 \\
Soda $\operatorname{ash}(\mathrm{g} / \mathrm{L})$ & 10 & 12 & 16 & 18 & 18 \\
\hline
\end{tabular}

\section{Soaping Treatment}

After dyeing the soaping off operation was conducted at a liquor ratio of 50:1 for 15 minutes using $2 \mathrm{~g} / \mathrm{L}$ non-ionic detergent (Luton 500, Dalton UK Company) in a rotary infrared laboratory dyeing machine.

\section{Dye Exhaustion}

In exhaust dyeing, all the material contacts all the dye liquor and the fibre absorbs the dyes. The dye concentration in the bath therefore gradually decreases. The degree of dye bath exhaustion is therefore a function of time describes the rate and extent of the dyeing process. For a single dye, the 
exhaustion (E\%) is defined as the mass of the dye taken up by the material divided by the total initial mass of dye in the bath and can be calculated according to Eq. 1.

$$
\mathrm{E} \%=\frac{\mathrm{M}_{1}-\mathrm{M}_{2}}{\mathrm{M}_{1}} \times 100
$$

Where $M_{1}$ is the initial mass of dye in dye bath, and the difference of $\left(M_{1-} M_{2}\right)$ is the mass of dye taken up by the material.

\section{Dye Fixation}

Proportion of dye remaining on the fiber at the end of the process is relative to the amount originally available. Fixation is basically how much the dye reacts with the fabric. It depends how much dye fixed on fabric considering all washes. Fixation ratio $(\mathrm{F} \%)$ was calculated by measuring $\mathrm{K} / \mathrm{S}$ value before soaping off treatment and after soaping off treatment (Eq.2).

$$
\mathrm{F} \%=\frac{(\mathrm{K} / \mathrm{S})_{2}}{(\mathrm{~K} / \mathrm{S})_{1}} \times 100
$$

Where $(\mathrm{K} / \mathrm{S})_{1}$ is the $\mathrm{K} / \mathrm{S}$ value before soaping off, $(\mathrm{K} / \mathrm{S})_{2}$ is the K/S value after soaping off.

\section{Application of UV Absorber}

Cationic CANFIX SUN UV absorber was applied on reactive dyed samples by exhaust method $4 \%$ o.w.f at a liquor ratio of $1: 50$ for $30 \mathrm{~min}$ at $40^{\circ} \mathrm{C}$. This operation was conducted in rotary infrared laboratory dyeing machine. After treatment the samples were washed and then air dried.

\section{Light Fastness Testing}

Light fastness testing of the dyed fabric samples without UV absorber application and after UV absorber application was done in Q-SUN Xe-1 machine where these samples were exposed separately for $1,6,12,24$ and $36 \mathrm{hrs}$ at $40^{\circ} \mathrm{C}$ under artificial light source (day light B01) equipped with xenon arch lamp whose wave length is similar to sun light. The change was compared with original specimen and assessed with Data Color CIE Lab dE value.

\section{Results and Discussion}

\section{Dye Exhaustion and Fixation}

Liyuan Yellow F S dye show not very good exhaustion but good fixation properties in Fig.2. The good fixation performance of Liyaun Yellow F S dye is contributed by the flourene base structure, which has very good reactivity to form covalent bond with cellulosic fiber.

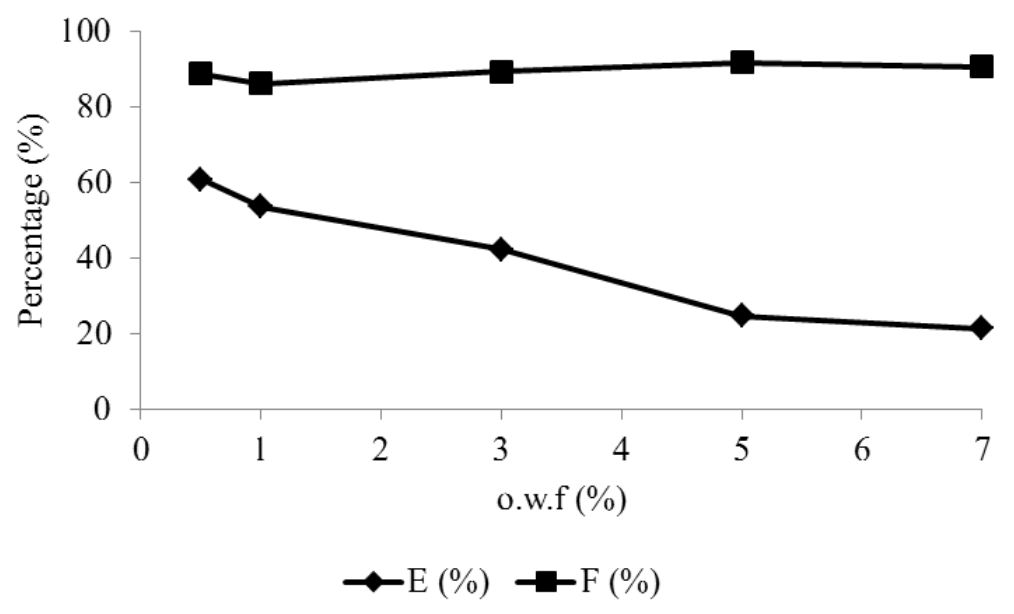

Fig.2 Exhaustion and Fixation with dye o.w.f \% 


\section{Light Fastness}

The light fastness of Yellow F S dyed samples were studied without application of UV absorber after exposing these samples to light for 1, 6, 12, 24 and $36 \mathrm{hrs}$. The shade change was studied by finding $\mathrm{dE}$ by comparing covered portion and exposed portion of dyed sample with Data Color CIE Lab $\mathrm{dE}$ value. Colour difference was measured by keeping dyed sample as standard and faded sample as batch. In Fig. 3, it shows that the fading of colour increases as duration of exposure of dyed sample to light increases like after $1 \mathrm{hr}$ exposure as shown in figure $\mathrm{dE}$ (total change in color) is lowest and then increases gradually for 6, 12, 24 hrs exposure and dE value is highest after $36 \mathrm{hrs}$ exposure.

As shown in graph light fastness of light shade is very poor as $\mathrm{dE}$ value of $0.5 \%$ o.w.f is high $\mathrm{dE}$ 1.55 and as shade depth increases $\mathrm{dE}$ (7\% o.w.f $\mathrm{dE}$ 0.55) decreases that means change in color is very less for dark shades it means as light fastness becomes good as shade depth increases and it is best for dark shades as shown in graph for 7\% o.w.f shade dE value has no change after 1, 6 and 12 hrs exposure and it shows a merged point in graph after 1, 6 and $12 \mathrm{hrs}$ exposure and from graph we can see very less change in $\mathrm{dE}$ value after 24 and $36 \mathrm{hrs}$ exposure.

Darker coloured fabrics can offer more protection than lighter coloured fabrics [9].Fabric dyed with dark shade has maximum protection from ultraviolet radiation. The clothing which are specially designed for ultraviolet protection these are generally dyed with high concentration of dyes these high concentration disrupt ultraviolet light.Some of the vinyl sulphone dyes and monochlorotriazine dyes have ultraviolet radiation absorption characteristics, which also increase with the concentration The smaller the surface area the smaller the degree of destruction of the dye molecules. The dye in low dye concentration, the dye on the fiber was highly dispersed state .The extent of the destruction and suffering outside chance of significantly larger so the light fastness in light shades is low as shown in graph also [10].

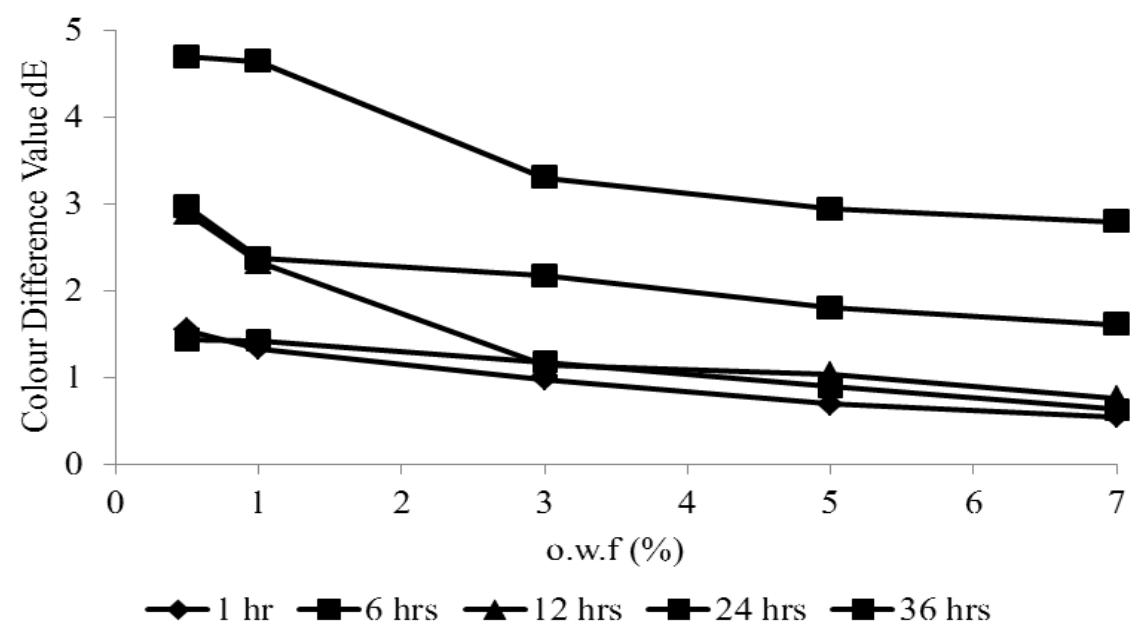

Fig.3 Fading rate curve of Liyuan Yellow F S without UV absorber application

\section{Effect of UV Absorbers on Light Fastness}

One of the basic requirement of application of UV absorber is it must not change the colour of dyed samples but here UV absorber application brought changes in bluer or brighter directions but no change in shade depth occurred after UV absorber application. The rate fading curves trend without UV absorber application and after UV absorber application is same that the colour fading increases as the time exposure increases. The graph in the Fig. 4 shows that the fading rate curves are closer to each other than in the Fig. 3 that means change in colour decreases after UV absorber application even in light shades we can see the curves after 1,6 and $12 \mathrm{hrs}$ exposure are almost same (merged curves in graph) that means no change in colour after UV absorber application. After 24 and 36 hrs exposure little change in colour and curve also less incline as compared to curves in Fig. 
3 that means after UV absorber application light shade light fastness improves as dark shades already having good light fastness and it shows almost straight line.

The function of UV absorbers is to absorb incident UV light, decrease the exposure of dyes to the UV lights and, therefore, improve the light fastness of the colour. UV absorbers forms agglomerates that decreases the coverage of UV light absorbs on fiber surfaces, therefore, decreases the exposure of dyes molecules to UV light[5].

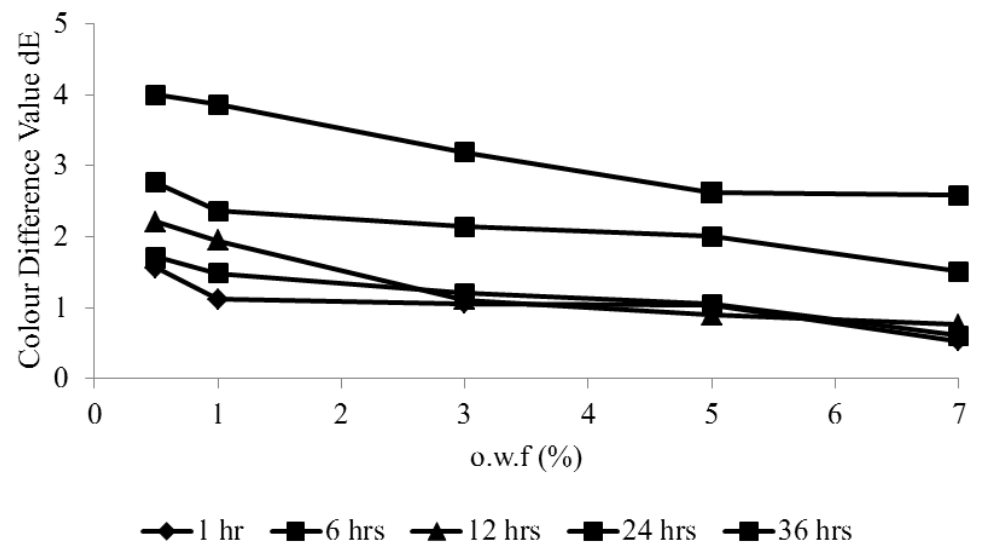

Fig.4 Fading rate curve of Liyuan Yellow F S after UV absorber application

The light fastness (36 hrs exposure) of dyed samples (4\% o.w.f UV absorber) has been studied and the results was shown in Fig.4. The graph shows improvement in light fastness in light shades and dark shades equally well and trend in graph shows almost straight line from left to right not slope from left to right in Fig.3 for un treated dyed samples. UV absorber is effective in improving light fastness from light to dark shades as line in graph is almost straight line it shows a little less improvement in light shades and more improvement in dark shades why little less improvement in light shades this is good scope for future research.

\section{Conclusion}

Fading rate curve for colour difference measurements $(\mathrm{dE})$ of Liyuan Yellow F S dyed samples show that colour fading increases gradually the time of exposure of dyed sample increases. The application of UV absorbers gives the colour change of reactive dyed samples in blue and brighter direction. After the UV absorber application light fastness property of reactive dyes improves. Fading rate curves of UV absorber applied dyed samples are alike little straight and close to each other, it shows improvement in light fastness property. Light fastness property of the reactive dyes depends on shade depth. As shade depth increases light fastness is good, but light fastness is poor for very light shade without UV absorber application. After UV absorber application light fastness property of light shades show improvement.

\section{Acknowledgements}

This work was supported by the China National Textile and Apparel Council (2013 "Textile Vision" Applied Basic Research) and a grant from Hubei Province Science and Technology Support Program (Project 2013BAA043).

\section{References}

[1]N. Kuramato, A. Peter, H. Freeman, Physico-chemical principle of colour chemistry, first ed., Blackie A\&P, London, 1996.

[2]J. Oakes, Photofading of textile dyes, Review of Progress in Coloration and Related Topics. 31 (2001) 21-28. 
[3]A.G. Brenton, R.P. Morgan, J.H. Beynon, Unimolecular ion decomposition, Annual Review of Physical Chemistry. 30 (1979) 51-78.

[4]N. Genc, E. Can-Dogan, Photooxidation: A decolorization procedure and a pre-treatment step for biodegradation of reactive azo dye, Polish Journal of Environmental Studies. 15 (2006) 73-79.

[5]Y. Yang, V. Naarani, Improvement of the lightfastness of reactive inkjet printed cotton, Dyes and Pigments. 74 (2007) 154-160.

[6]G. Reinert, F. Fuso, Stabilisation of textile fibres against ageing, Review of Progress in Coloration and Related Topics. 27 (1997) 32-41.

[7]G. Reinert, E. Schmidt, R. Hilfiker, Facts about the application of uv absorbers on textiles, Melliand Textilberichte International Textile Reports. 75 (1994) E151-E151.

[8]P. Thiagarajan, G. Nalankilli, Improving light fastness of reactive dyed cotton fabric with antioxidant and uv absorbers, Indian Journal of Fibre \& Textile Research. 38 (2013) 161-164.

[9]B.R. Das, Uv radiation protective clothing, Open Textile Journal. 3 (2010) 14-21.

[10]H. Cui, Improve the light fastness of reactive dyes (2), Yin ran. 13 (2005) 14-17. 